\title{
Hidatidosis pulmonar: Hallazgos y tratamiento quirúrgico en quistes complicados versus no complicados
}

\author{
Roberto González L., ${ }^{1,2}$, Alejandra Riquelme U. ${ }^{3}$, Matías Ávalos T. ${ }^{5}$, \\ Rodrigo Reyes M. ${ }^{1,2}$, Enrique Seguel S. ${ }^{1,2}$, Aleck Stockins L. ${ }^{1,2}$, \\ Andrés Jadue T. ${ }^{1}$, Loreto Spencer L. ${ }^{4}$, Claudio Toloza A. ${ }^{5}$, \\ Sebastián Barra M. ${ }^{5}$, Felipe Alarcón O. ${ }^{5}$ y Emilio Alarcón C. ${ }^{1,2}$
}

\section{Pulmonary hydatidosis: Findings and surgical treatment in complicated versus uncomplicated cysts}

Background: Human hydatidosis is a prevalent zoonosis in our environment, caused mainly by the Echinococcus granulosus parasite. The most common complications in the lung are cyst rupture and infection. Aim: To describe and compare clinical characteristics, treatment, morbidity and mortality of patients hospitalized for complicated versus uncomplicated hydatid pulmonary cyst (HPC). Materials and Method: Longitudinal analytical study. Period January 1973 - December 2017 at the Regional Clinical Hospital of Concepción "Dr. Guillermo Grant Benavente", Chile. Database, prospective protocols and medical records were reviewed. Microsoft Exce ${ }^{\mathbb{B}}$ spreadsheet and SPSS2 $4^{\mathbb{Q}}$ program with chi square and Student's t-test were used. It was considered significant $p<0.05$. Results: Total 364 episodes of HPC, complicated 179 (49.2\%) versus uncomplicated 185 (50.8\%). Average age $36.4 \pm 18.9$ versus $32.4 \pm 19.1$ years, male $114(63.7 \%)$ versus $107(57.8 \%)$, respectively. A statistically significant difference was found in: symptomatology cough $147(82.1 \%)$ versus $120(64.9 \%)$, hemoptysis $93(52.0 \%)$ versus $45(24.3 \%)$, respectively; HPC right $115(64.2 \%)$ versus 99 (53.5\%), respectively; resective cyst surgery $139(77.7 \%)$ versus $167(90.3 \%)$, reoperation $22(12.3 \%)$ versus $8(4.3 \%)$, respectively; morbidity $47(26.3 \%)$ versus $30(16.2 \%)$, mortality $6(3.4 \%)$ versus $0(0 \%)$, average postoperative hospital stay $16.1 \pm 14.3$ versus 10.7 \pm 7.7 days, recurrence $15(8.4 \%)$ versus $13(7.0 \%)$, respectively. Conclusions: Approximately half of the HPCs are complicated. Complicated HPCs present statistically significant differences in their clinical, treatment, morbidity, mortality and postoperative hospital stay.

Key words: echinococcosis; pulmonary; thoracic surgery; zoonoses; lung diseases; operative surgical procedures.

\section{Resumen}

Introducción: La hidatidosis humana es una zoonosis prevalente en nuestro medio, causada principalmente por el parásito Echinococcus granulosus. Las complicaciones más frecuentes en el pulmón son la rotura y la infección del quiste. Objetivos: Describir y comparar características clínicas, tratamiento, morbilidad y mortalidad de los pacientes hospitalizados por quiste hidatídico pulmonar (QHP) complicado versus no complicado. Materiales y Método: Estudio analítico longitudinal. Período enero de 1973 - diciembre de 2017 en Hospital Clínico Regional de Concepción "Dr. Guillermo Grant Benavente", Chile. Revisión de base de datos, protocolos de QHP y fichas clínicas. Se utilizó planilla Microsoft Excel ${ }^{\mathbb{B}}$ y programa SPSS24®, con función chi cuadrado y $t$ de Student. Se consideró significativo $\mathrm{p}<0,05$. Resultados: Total 364 episodios de QHP, complicados 179 (49,2\%) versus no complicados $185(50,8 \%)$. Edad promedio $36,4 \pm 18,9$ versus 32,4 $\pm 19,1$ años, hombres $114(63,7 \%)$ versus 107 (57,8\%), respectivamente. Se encontró diferencia estadísticamente significativa en: sintomatología tos $147(82,1 \%)$ versus $120(64,9 \%)$, hemoptisis $93(52,0 \%)$ versus 45 (24,3\%), respectivamente; QHP derecho $115(64,2 \%)$ versus $99(53,5 \%)$, respectivamente; cirugía conservadora $139(77,7 \%)$ versus $167(90,3 \%)$, reoperaciones $22(12,3 \%)$ versus
${ }^{1}$ Centro Cardiovascular, Hospital Clínico Regional de Concepción Dr. Guillermo Grant Benavente. Concepción, Chile.

Departamento de Cirugía, Facultad de Medicina

Universidad de Concepción. Concepción, Chile.

${ }^{3}$ Servicio de Cirugía,

Hospital Clínico Regional de Concepción Dr. Guillermo Grant Benavente. Concepción, Chile.

${ }^{4}$ Unidad de Anatomía Patológica, Hospital Clínico Regional de Concepción Dr. Guillermo Grant Benavente. Concepción, Chile. ${ }^{5}$ Estudiante de Medicina, Facultad de Medicina, Universidad de Concepción. Concepción, Chile.

Recibido el 19 de julio de 2019 y aceptado para publicación el 7 de septiembre de 2019.

Correspondencia a: Dr. Roberto González L. rgonzalezlagos@udec.cl 
$8(4,3 \%)$, respectivamente; morbilidad $47(26,3 \%)$ versus $30(16,2 \%)$, mortalidad $6(3,4 \%)$ versus $0(0 \%)$, estadía hospitalaria postoperatoria promedio $16,1 \pm 14,3$ versus $10,7 \pm 7,7$ días, recidivas $15(8,4 \%)$ versus 13 (7,0\%), respectivamente. Discusión: Aproximadamente la mitad de los QHP son complicados. Los QHP complicados presentan diferencias estadísticamente significativas en su clínica, tratamiento, morbilidad, mortalidad y estadía hospitalaria postoperatoria.

Palabras clave: equinococosis pulmonar; cirugía torácica; zoonosis; enfermedades pulmonares; procedimientos quirúrgicos operativos.

\section{Introducción}

La hidatidosis es una zoonosis producida en nuestro medio por el parásito Echinococcus granulosus. Es endémica en distintos lugares en donde habita el ganado y se describe más frecuentemente en países mediterráneos, Oriente Medio, Sudamérica, Australia y Nueva Zelanda ${ }^{1-5}$.

Los quistes hidatídicos se desarrollan prácticamente en cualquier órgano, siendo los más frecuentes el hígado y el pulmón ${ }^{6,7}$.

Los quistes hidatídicos pulmonares (QHP) se complican al menos en un $50 \%$, siendo las complicaciones más frecuentes la rotura e infección ${ }^{8}$. En nuestro país existen escasas y poco numerosas series recientes que presenten el problema de la hidatidosis pulmonar.

Nuestros objetivos son describir y comparar características clínicas, tratamiento, morbilidad y mortalidad de los pacientes hospitalizados por QHP complicado versus no complicado.

\section{Materiales y Método}

Se realizó un estudio analítico longitudinal de los pacientes hospitalizados por QHP en el Hospital Clínico Regional "Dr. Guillermo Grant Benavente" de Concepción, Chile, tratados por el equipo de Cirugía Cardiotorácica, en el período comprendido entre enero de 1973 y diciembre de 2017.

La información se obtuvo a partir de protocolos prospectivos de QHP realizados durante la estadía del paciente en el hospital, revisión de base de datos del equipo de Cirugía Cardiotorácica, protocolos quirúrgicos, fichas clínicas, registros de anatomía patológica y certificados de defunción del Registro Civil e Identificación de Chile.

Se ordenaron los datos mediante planilla de $\mathrm{Mi}$ crosoft Excel ${ }^{\mathbb{B}}$ y se realizó análisis estadístico con el programa SPSS24 ${ }^{\circledR}$, con función chi cuadrado para las variables cualitativas y con la función $t d e$ Student para las variables cuantitativas. Se consideró significativo $\mathrm{p}<0,05$.
Se describe y se compara edad, sexo, características clínicas, diagnóstico, ubicación y características del quiste, tratamiento, estadía postoperatoria, morbilidad, mortalidad, seguimiento y recidiva.

Se consideraron QHP complicados aquellos que se encontraban al momento del diagnóstico con evidencia de infección, con rotura al árbol bronquial, con rotura a la cavidad pleural y/o membrana retenida (pudiendo presentar más de una complicación cada quiste).

El estudio y análisis de las hospitalizaciones por hidatidosis torácica fue aprobado por el comité de ética de nuestra institución.

\section{Resultados}

El total de episodios de QHP tabulados con datos completos fue de 364, de ellos 185 (50,8\%) fueron no complicados y $179(49,2 \%)$ complicados. Las complicaciones más frecuentes fueron la infección y la rotura al árbol bronquial. De los QHP infectados, $3(3,1 \%)$ se presentaron como abscesos (Tabla 1$)$.

La edad promedio fue mayor en los QHP complicados que en los no complicados, no existiendo diferencias en el sexo ni residencia rural al momento del diagnóstico entre ambos grupos (Tabla 2).

Al momento del diagnóstico, la sintomatología más frecuente en ambos grupos fue la tos y el dolor torácico. Se encontraron diferencias estadísticamen-

Tabla 1. Condición de los quistes hidatídicos pulmonares al momento del diagnóstico

\begin{tabular}{|lc|}
\hline & Total episodios QHP(364) \\
\hline Quiste no complicado & $185(50,8 \%)$ \\
Quiste complicado & $179(49,2 \%)$ \\
Infección & $96(26,4 \%)$ \\
Rotura al árbol bronquial & $81(22,3 \%)$ \\
Membrana retenida & $58(15,9 \%)$ \\
Rotura a la cavidad pleural & $27(7,4 \%)$ \\
\hline
\end{tabular}

QHP: Quiste hidatídico pulmonar. 
te significativas entre los QHP complicados y no complicados en la tos, hemoptisis o expectoración hemoptoica y fiebre. Relataron vómica hidatídica 98 $(26,9 \%)$ casos. Fueron asintomáticos en su presentación $2(1,1 \%)$ QHP complicados y $26(14,1 \%)$ QHP no complicados, con diferencia estadísticamente significativa entre ellos (Tabla 3 ).

Dentro del estudio radiológico la mayoría de las radiografías resultaron sospechas de hidatidosis pulmonar en los QHP no complicados con diferencia estadísticamente significativa versus los QHP complicados. La primera tomografía computada (TC) de tórax en nuestra serie se realizó en enero de 1991, ya que no se disponía de este examen anteriormente en nuestro hospital (Figura 1). En relación a la detección de eosinofilia en el hemograma, no hubo diferencia según la condición del QHP. Se realizó serología (enzimoinmunoanálisis de adsorción para inmunoglobulina $\mathrm{E}$ y para inmunoglobulina $\mathrm{G}$, inmunoelectrofóresis para arco 5 de Caprón y hemoaglutinación indirecta) en $65(36,3 \%)$ casos de QHP complicados y en $53(28,6 \%)$ casos de QHP no complicados. Se observó mayor porcentaje de serología positiva en QHP complicados que en QHP no complicados: 55 (84,6\%) versus 29 (54,7\%), respectivamente, con $\mathrm{p}<0,001$ (Tabla 4 ).
En cuanto a la caracterización de los QHP, en ambos grupos fueron predominantemente únicos y uniloculares, sin diferencias significativas. El diámetro promedio del quiste complicado fue $8,4 \mathrm{~cm}$ y del no complicado $9,7 \mathrm{~cm}$, con diferencia significativa. En cuanto a su localización, no existieron diferencias estadísticamente significativas, exceptuando en el lóbulo inferior izquierdo en donde se encontraron mayormente quistes no complicados. No existieron diferencias en cuanto a presentación bilateral al ingreso y asociación con quistes en otras localizaciones (Tabla 5).

Con respecto al tratamiento, se realizó cirugía en $354(97,3 \%)$ casos. Los 10 episodios que no se operaron fueron principalmente por negativa del paciente. Se realizaron cirugías conservadoras (resectivas quísticas) más frecuentemente en los QHP no complicados, con diferencias significativas. Dentro de estas, encontramos las quistectomías con capitonaje y sin capitonaje, encontrándose mayor proporción de quistectomía con capitonaje en los QHP no complicados, con diferencia estadísticamente significativa. Las cirugías radicales (anatómicas) fueron más frecuentes en los QHP complicados, sin alcanzar diferencias significativas. Dentro de las otras cirugías realizadas, se encuentran decortica-

Tabla 2. Edad, sexo y ruralidad de los pacientes con quistes hidatídicos pulmonares según condición

\begin{tabular}{|lccc|}
\hline & QHP complicados (179) & QHP no complicados (185) & p \\
Edad (en años) & & & $0,045^{*}$ \\
$\quad$ Promedio & $36,4 \pm 18,9$ & $32,4 \pm 19,1$ & 0,253 \\
Sexo & & & \\
$\quad$ Hombre & $114(63,7 \%)$ & $107(57,8 \%)$ & 0,661 \\
$\quad$ Mujer & $65(36,3 \%)$ & $78(42,2 \%)$ & $129(69,7 \%)$ \\
Domicilio rural** & $121(67,6 \%)$ & & \\
\hline
\end{tabular}

QHP: Quiste hidatídico pulmonar; *Estadísticamente significativo; **Al momento del diagnóstico.

Tabla 3. Sintomatología de los pacientes según condición del quiste hidatídico pulmonar al momento del diagnóstico

\begin{tabular}{|lccc|}
\hline Síntomas y signos & QHP complicados (179) & QHP no complicados (185) & p \\
\hline Tos & $147(82,1 \%)$ & $120(64,9 \%)$ & $<0,001^{*}$ \\
Dolor torácico & $107(59,8 \%)$ & $109(58,9 \%)$ & 0,868 \\
Disnea & $81(45,3 \%)$ & $66(35,7 \%)$ & 0,063 \\
Hemoptisis y/o expectoración hemoptoica & $93(52,0 \%)$ & $45(24,3 \%)$ & $<0,001^{*}$ \\
Fiebre & $52(29,1 \%)$ & $35(18,9 \%)$ & $0,023^{*}$ \\
Asintomáticos & $2(1,1 \%)$ & $26(14,1 \%)$ & $<0,001^{*}$ \\
\hline
\end{tabular}

QHP: Quiste hidatídico pulmonar; *Estadísticamente significativo. 


\section{ARTÍ́CULO ORIGINAL}
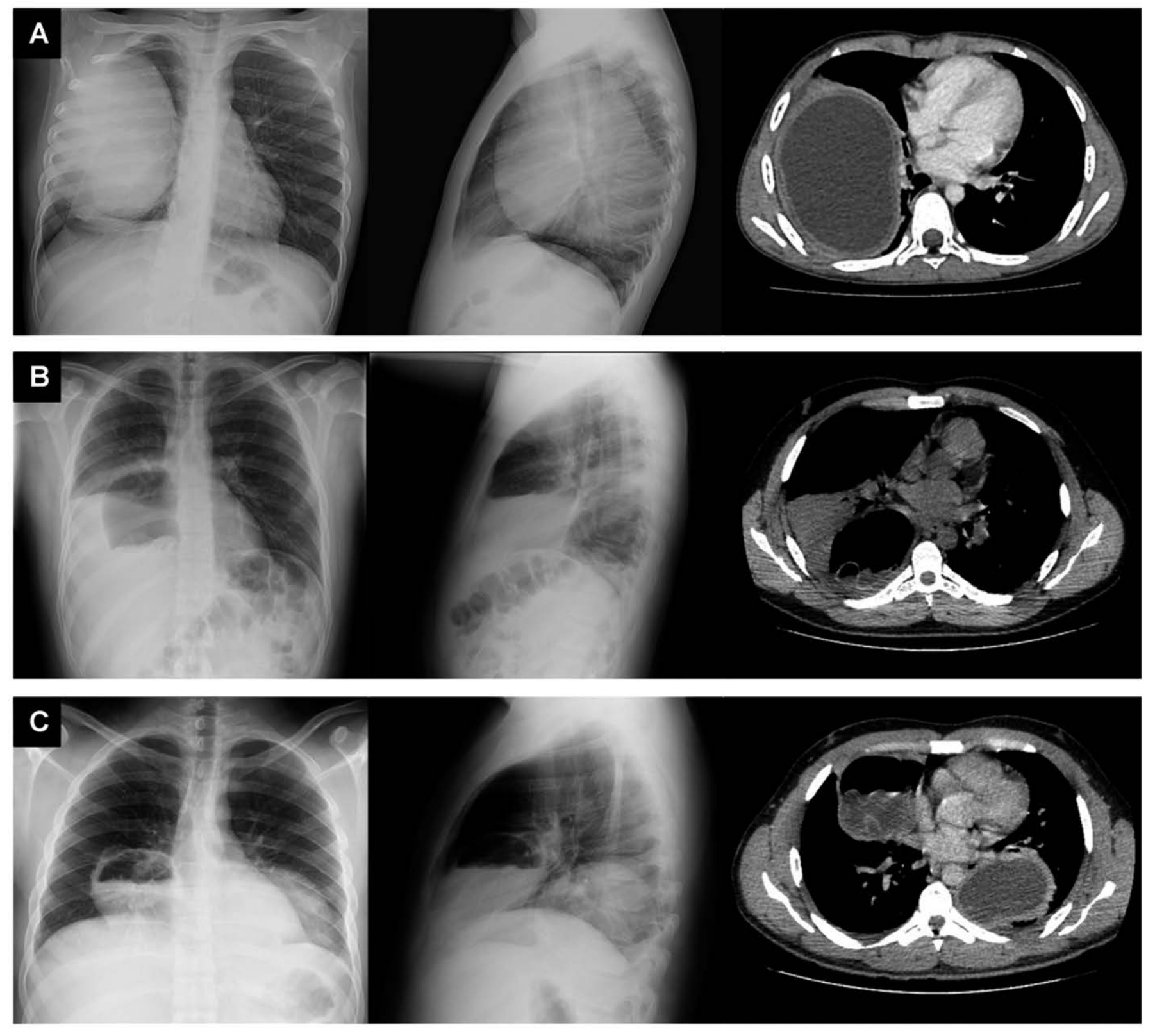

Figura 1. Estudio imagenológico de quiste hidatídico pulmonar. Radiografía de tórax postero-anterior, lateral y tomografía computada de tórax que muestran A: Quiste hidatídico pulmonar (QHP) no complicado derecho. B: QHP complicado derecho en que se observa membrana hidatídica en cavidad y atelectasia lobar. C: QHP complicado derecho y QHP no complicado retrocardíaco izquierdo.

ciones, reparaciones diafragmáticas, pleurectomía, cierre de fístulas, etcétera, siendo más frecuentes en los QHP complicados con diferencias significativas (Tabla 6).

Se encontró también significancia estadística en morbilidad, reoperaciones, mortalidad y estadía postoperatoria, siendo menor en los QHP no complicados. Las complicaciones más frecuentes fueron la fístula broncopleural y el empiema, ocurriendo ambas en su mayoría en QHP complicados. Destacamos que no existió mortalidad en los QHP no complicados versus un 3,4\% en los QHP complicados (Tabla 7).

Se realizó seguimiento por un período promedio 
Tabla 4. Estudio diagnóstico de los pacientes con quistes hidatídicos pulmonares según condición

\begin{tabular}{|lccc|}
\hline & QHP complicados (179) & QHP no complicados (185) & p \\
\hline Radiografías de tórax sospechosas de QHP & $142(79,3 \%)$ & $168(90,8 \%)$ & $0,002 *$ \\
TC tórax realizado & $77(43,0 \%)$ & $74(40,0 \%)$ & 0,559 \\
Eosinofilia & $90(50,6 \%)$ & $76(41,5 \%)$ & 0,085 \\
Serología realizada & $65(36,3 \%)$ & $53(28,6 \%)$ & 0,118 \\
$\quad$ Serología positiva & $55(84,6 \%)$ & $29(54,7 \%)$ & $<0,001 *$ \\
\hline
\end{tabular}

QHP: Quiste hidatídico pulmonar; TC: Tomografía computada; *Estadísticamente significativo.

Tabla 5. Características, ubicación, bilateralidad y quistes asociados de los quistes hidatídicos pulmonares según condición

\begin{tabular}{|c|c|c|c|}
\hline & QHP complicados (179) & QHP no complicados (185) & $\mathbf{p}$ \\
\hline $\begin{array}{l}\text { Características de los quistes } \\
\text { Único } \\
\text { Múltiple } \\
\text { Quiste unilocular } \\
\text { Tamaño promedio quiste }(\mathrm{cm})\end{array}$ & $\begin{array}{r}136(76,0 \%) \\
43(24,0 \%) \\
158(88,3 \%) \\
8,4 \pm 4,5\end{array}$ & $\begin{array}{r}151(81,6 \%) \\
34(18,4 \%) \\
173(93,5 \%) \\
9,7 \pm 5,1\end{array}$ & $\begin{array}{l}0,187 \\
0,187 \\
0,081 \\
0,014 *\end{array}$ \\
\hline $\begin{array}{l}\text { Lóbulos superiores } \\
\text { Izquierdo } \\
\text { Derecho }\end{array}$ & $\begin{array}{l}80(44,7 \%) \\
41(22,9 \%) \\
40(22,3 \%)\end{array}$ & $\begin{array}{l}68(36,8 \%) \\
42(22,7 \%) \\
27(14,6 \%)\end{array}$ & $\begin{array}{l}0,123 \\
0,963 \\
0,056\end{array}$ \\
\hline Lóbulo medio & $19(10,6 \%)$ & $18(9,7 \%)$ & 0,780 \\
\hline $\begin{array}{l}\text { Lóbulos inferiores } \\
\text { Izquierdo } \\
\text { Derecho }\end{array}$ & $\begin{array}{l}99(55,3 \%) \\
30(16,8 \%) \\
70(39,1 \%)\end{array}$ & $\begin{array}{r}119(64,3 \%) \\
55(29,7 \%) \\
65(35,1 \%)\end{array}$ & $\begin{array}{l}0,079 \\
0,003 * \\
0,433\end{array}$ \\
\hline Bilateral al ingreso & $13(8,2 \%)$ & $21(12,5 \%)$ & 0,200 \\
\hline $\begin{array}{l}\text { Quiste asociado } \\
\text { Hepático } \\
\text { Otros }\end{array}$ & $\begin{array}{l}43(24,0 \%) \\
36(20,1 \%) \\
12(6,7 \%)\end{array}$ & $\begin{array}{r}42(22,7 \%) \\
36(19,5 \%) \\
9(4,9 \%)\end{array}$ & $\begin{array}{l}0,766 \\
0,876 \\
0,452\end{array}$ \\
\hline Tránsito hepato-torácico & $7(3,9 \%)$ & $3(1,6 \%)$ & 0,182 \\
\hline
\end{tabular}

QHP: Quiste hidatídico pulmonar; *Estadísticamente significativo.

Tabla 6. Tratamiento de los pacientes con quistes hidatídicos pulmonares según condición

\begin{tabular}{|lccc|}
\hline & QHP complicados (179) & QHP no complicados (185) & p \\
\hline Tratamiento quirúrgico & $172(96,1 \%)$ & $182(98,4 \%)$ & 0,182 \\
Cirugía conservadora & $139(77,7 \%)$ & $167(90,3 \%)$ & $0,001^{*}$ \\
Quistectomía con capitonaje & $87(48,6 \%)$ & $120(64,9 \%)$ & $0,002^{*}$ \\
Quistectomía sin capitonaje & $52(29,1 \%)$ & $47(25,4 \%)$ & 0,435 \\
Cirugía radical & $23(12,9 \%)$ & $15(8,1 \%)$ & 0,139 \\
Lobectomía & $13(7,3 \%)$ & $10(5,4 \%)$ & 0,467 \\
Segmentectomía atípica & $7(3,9 \%)$ & $5(2,7 \%)$ & 0,519 \\
Neumonectomía & $3(1,7 \%)$ & $0(0 \%)$ & 0,077 \\
Otra cirugía & $31(17,3 \%)$ & $2(1,1 \%)$ & $<0,001^{*}$ \\
Lateralidad cirugía & & & $0,038^{*}$ \\
Derecha & $115(64,2 \%)$ & $99(53,5 \%)$ & 0,081 \\
Izquierda & $68(38,0 \%)$ & $87(47,0 \%)$ & $0,017^{*}$ \\
Tratamiento farmacológico** & $53(29,6 \%)$ & $35(18,9 \%)$ & \\
\hline
\end{tabular}

QHP: Quiste hidatídico pulmonar; *Estadísticamente significativo; **Asociado. 
Tabla 7. Morbilidad, reoperaciones, mortalidad y estadía postoperatoria de pacientes con quistes hidatídicos pulmonares según condición

\begin{tabular}{|lccc|}
\hline & QHP complicados & QHP no complicados & p \\
Morbilidad & $47(26,3 \%)$ & $30(16,2 \%)$ & $0,019^{*}$ \\
$\quad$ Fístula broncopleural & $30(16,8 \%)$ & $8(4,3 \%)$ & $<0,001^{*}$ \\
Empiema & $9(5,0 \%)$ & $2(1,1 \%)$ & $0,028^{*}$ \\
Hemotórax & $6(3,4 \%)$ & $2(1,1 \%)$ & 0,140 \\
Otros & $13(7,3 \%)$ & $12(6,5 \%)$ & 0,770 \\
Reoperaciones & $22(12,3 \%)$ & $8(4,3 \%)$ & $0,006^{*}$ \\
Mortalidad & $6(3,4 \%)$ & $0(0 \%)$ & $0,012^{*}$ \\
Estadía postoperatoria (días) & & $10,7 \pm 7,7$ & $<0,001^{*}$ \\
$\quad$ Promedio & $16,1 \pm 14,3$ & 8 & \\
\hline
\end{tabular}

QHP: Quiste hidatídico pulmonar; *Estadísticamente significativo.

Tabla 8. Seguimiento y recidiva de los pacientes con quistes hidatídicos pulmonares según condición

\begin{tabular}{|lccc|}
\hline & QHP complicados (179) & QHP no complicados (185) & p \\
\hline Seguimiento promedio (meses) & $296,8 \pm 163,5$ & $289,7 \pm 159,9$ & 0,674 \\
Recidiva & $15(8,4 \%)$ & $13(7,0 \%)$ & 0,628 \\
\hline
\end{tabular}

QHP: Quiste hidatídico pulmonar.

de 293,2 meses (24,4 años), con un rango de 10,9 a 550,4 meses. No se observó diferencia en la recidiva entre ambos grupos (Tabla 8).

\section{Discusión}

Todos los QHP tienen el riesgo de complicarse. Las complicaciones más frecuentes corresponden a la rotura y la infección. Los QHP complicados en nuestra serie tienen mayor morbilidad, mortalidad y estadía hospitalaria que los no complicados. Aproximadamente la mitad de nuestros casos se encontraban complicados al momento del diagnóstico, pudiendo presentar más de una complicación simultáneamente. En conjunto, la rotura del quiste, ya sea hacia la vía aérea y/o hacia la cavidad pleural, es la complicación más frecuente. En la literatura las tasas de quistes complicados al momento del diagnóstico oscilan entre 33\%-59\% $0^{1,3,4,8-10}$.

La hidatidosis pulmonar se presenta clásicamente en hombres menores de 40 años y de procedencia rural ${ }^{1,3-5,8,10-13}$. Puede ser asintomática, siendo más frecuente este tipo de presentación en los QHP no complicados. En nuestra serie los pacientes asintomáticos fueron un 14,1\% en QHP no complicados.

Los QHP no complicados suelen presentarse con tos, dolor torácico y disnea ${ }^{1,2}$. Por su parte, los
QHP complicados rara vez son asintomáticos, ya que si están infectados se manifestarán en la mayor parte de los casos con fiebre, si se rompe hacia los bronquios se podrá observar la vómica hidatídica, expectoración hemoptoica y/o hemoptisis, y si se rompen hacia la pleura se expresarán con la clínica de ocupación pleural y/o posterior empiema. En nuestra serie, los pacientes con QHP complicados presentaron más tos, hemoptisis y fiebre que los no complicados.

La mayoría de los QHP pueden ser diagnosticados con una radiografía de tórax ${ }^{14}$. En nuestra serie la sospecha radiológica fue mayor en los QHP no complicados, esto podría explicarse porque el quiste al romperse pierde sus signos imagenológicos característicos, dificultando de este modo el diagnóstico. En la actualidad se utilizan dos pruebas serológicas: enzimoinmunoanálisis de adsorción para inmunoglobulina G para tamizaje y Western blot para confirmación 7 , sin embargo, en nuestro estudio hemos incluido exámenes más antiguos ya que eran los disponibles al inicio de la serie. La serología resultó positiva en mayor proporción en los QHP complicados, una explicación aceptada a esto es que, al romperse el quiste, se genera una reacción sistémica con mayor cantidad de mediadores inflamatorios y una respuesta inmunológica de mayor envergadura, lo que se traduce en la positividad de estos exámenes. 
Los QHP pueden ser únicos o múltiples, siendo mucho más frecuente que sean únicos ${ }^{10,11}$, sin diferencias entre los complicados y no complicados. Los QHP complicados fueron de menor tamaño, ya que al romperse un quiste este pierde gran parte de su contenido y su forma característica, lo que determina menores diámetros. Los QHP se ubicaron en los lóbulos inferiores en más de la mitad de los casos, siendo el derecho más frecuente. En la literatura no existe un patrón único de distribución en los diferentes lóbulos, sin embargo, sí se observa un predominio de los lóbulos inferiores y del pulmón derecho ${ }^{1,3-5,10,11}$.

Los QHP pueden estar asociados a quistes en otras localizaciones, dentro de las cuales la más frecuente es la hepática. Otras ubicaciones en las que se describen son el bazo, corazón, pericardio, omentos, cerebro, mediastino, pared arteria pulmonar, riñones, peritoneo, huesos, páncreas, etcétera ${ }^{1,5,11,15,16}$. No encontramos diferencias en las ubicaciones de quistes asociados entre los QHP complicados y no complicados, así como tampoco en la presencia de quistes en tránsito hepato-torácico.

La cirugía es el tratamiento de elección de la hidatidosis pulmonar y dentro de las cirugías se deben priorizar las conservadoras o resectivas del quiste, es decir, aquellas en las que se realiza resección de la hidátide y parte de la adventicia. Dentro de las cirugías conservadoras encontramos la quistectomía con o sin capitonaje. El capitonaje es una técnica que consiste en aproximar los bordes de la cavidad quística residual mediante la utilización de suturas, con el objetivo de disminuir el tamaño de ésta.

Sin embargo, no siempre es posible realizar cirugías conservadoras y deben plantearse en esos casos cirugías radicales o anatómicas, es decir, con resección anatómica del parénquima pulmonar. Se describe una mayor necesidad de estas cirugías en los QHP complicados ya que estos suelen asociarse a mayor destrucción del parénquima circundante $\mathrm{e}^{3,8,13,17}$. Dentro de estas cirugías encontramos segmentectomías (típicas y atípicas), lobectomías y neumonectomías. Diversos estudios reportan necesidades de cirugía radical que oscilan entre 0\%-7\% para los QHP no complicados y entre 19\%-32\% para los complicados ${ }^{8,17}$. En nuestra serie, el tratamiento quirúrgico conservador se realizó en $84,1 \%$ de los casos, predominando en los no complicados. Por otro lado, las cirugías radicales se realizaron principalmente en QHP complicados, sin existir una diferencia estadísticamente significativa entre ambos grupos. Es importante aclarar que un paciente con hidatidosis puede presentar más de un quiste, por lo que en algunos casos se pueden realizar distintas cirugías en un mismo episodio.

Los QHP complicados tienen una mayor morbilidad y mortalidad postoperatoria que los no complicados $^{8,18}$. El daño al parénquima circundante, ya sea por la rotura o infección del quiste, conlleva una recuperación más lenta y aumenta la posibilidad a distintas complicaciones postoperatorias como fístulas broncopleurales, empiema, hemotórax, neumotórax, infecciones de herida operatoria, entre otras. Se describe morbilidad postoperatoria en $6,9 \%-39 \%{ }^{10,18,19}$ de los casos, Kuzucu y cols ${ }^{8}$ reportan una tasa de complicaciones en 8,8\% de los QHP no complicados y 39,4\% de los QHP complicados. Balci y cols ${ }^{17}$ describen un 25,4\% de morbilidad postoperatoria en QHP complicados en niños. En nuestra serie observamos un 26,3\% de morbilidad en los QHP complicados, versus un $16,2 \%$ en los no complicados, siendo las principales diferencias entre estos el desarrollo de fístulas broncopleurales y empiemas posteriores a la cirugía.

La mortalidad general reportada en otras series oscila entre $0 \%-2,3 \% 0^{1,3-5,10,12,13,18-21}$. En series de QHP complicados los valores ascienden a $4,7 \%{ }^{17}$. En nuestros pacientes la mortalidad global fue 1,6\%, siendo en su totalidad a expensas de los QHP complicados, con diferencia estadísticamente significativa al compararse con los no complicados.

La estadía postoperatoria también se ha descrito más prolongada en los QHP complicados ${ }^{8}$. Concordantemente con la literatura, en nuestra serie los QHP complicados tienen una estadía postoperatoria más prolongada que los no complicados.

La recidiva se debe probablemente a reinfección, ya que a pesar de las medidas y las diversas campañas sanitarias que se realizan, las características epidemiológicas no cambian significativamente al tratarse de una población cautiva, con pocas tasas de migración, lo que les continúa predisponiendo a una nueva infección. Se realizó un seguimiento promedio global de 293,2 meses (24,4 años), sin diferencia significativa en la recidiva según el estado del quiste. Probablemente esto se explique por las características epidemiológicas, más que por la condición del quiste al momento del diagnóstico. Dadas las características descritas de la población, es posible que exista un subdiagnóstico de las recidivas.

En conclusión, los QHP complicados tienen una mayor mortalidad, morbilidad y estadía postoperatoria que los no complicados, por lo que se reafirma la idea del diagnóstico y tratamiento oportuno de los QHP. 


\section{Responsabilidades éticas}

Protección de personas y animales. Los autores declaran que para esta investigación no se han realizado experimentos en seres humanos ni en animales.
Confidencialidad de los datos. Los autores declaran que en este artículo no aparecen datos de pacientes.

Conflictos de interés: no hay.

\section{Bibliografía}

1. Aydm A, Yurdakul Y, Coşkun I, Rüstem O, Argun S. Pulmonary hydatid disease: report of 100 patients. Ann Thorac Surg. 1977;23:145-51

2. Sokouti M, Golzari S, Aghdam B. Surgery of uncomplicated pulmonary hydatid cysts: capitonnage or uncapitonnage? Int J Surg. 2011;9:221-4.

3. Doğan R, Yüksel M, Cetin G, Süzer K, Alp M, Kaya S, et al. Surgical treatment of hydatid cyst of the lung: report on 1055 patients. Thorax 1989;44:192-9.

4. Turna A, Yilmaz M, Haciibrahimoğlu G, Kutlu C, Bedirhan M. Surgical treatment of pulmonary hydatid cysts: is capitonnage necessary? Ann Thorac Surg. 2002;74:191-5.

5. Burgos R, Varela A, Castedo E, Roda J, Montero C, Serrano S, et al. Pulmonary hydatidosis: surgical treatment and followup of 240 cases. Eur J Cardiothorac Surg. 1999;16:628-35.

6. González R, Reyes R, Riquelme A, Seguel E, Stockins A, Jadue A, et al. Hidatidosis cardíaca: tratamiento quirúrgico para recidiva de quiste hidatídico en ventrículo izquierdo. Rev Cir. 2019;71:266-9.

7. Pinto P. Diagnóstico, tratamiento y seguimiento de la hidatidosis. Rev Chil Cir. 2017;69:94-8.

8. Kuzucu A, Soysal Ö, Özgel M, Yologlu S. Complicated hydatid cysts of the lung: clinical and therapeutic issues. Ann Thorac Surg. 2004;77:1200-4.

9. Novick R, Tchervenkov C, Wilson J, Munro D, Mulder D. Surgery for thoracic hydatid disease: a North American experience. Ann Thorac Surg. 1987;43:681-6.

10. Baquerizo A, Muñoz W, Barrientos F. Hidatidosis pulmonar: experiencia en 1094 quistes. Rev Chil Cir. 1998;50:5028.

11. Dakak M, Genç O, Gürkök S, Gözübüyük A, Balkanli K. Surgical treatment for pulmonary hydatidosis (a review of 422 cases). J R Coll Surg Edinb. 2002;47:68992.

12. Ulkü R, Yilmaz H, Onat S, Ozçelik C. Surgical treatment of pulmonary hydatid cysts: report of 139 cases. Int Surg. 2006;91:77-81.

13. Xanthakis D, Efthimiadis M, Papadakis G, Primikirios N, Chassapakis G, Roussaki A, et al. Hydatid disease of the chest. Thorax 1972;27:517-28.

14. Garg M, Sharma M, Gulati A, Gorsi U, Aggarwal A, Agarwal R, Khandelwal
N. Imaging in pulmonary hydatid cysts. World J Radiol. 2016;8:581-7.

15. Orhan G, Ozay B, Tartan Z, Kurc E, Ketenci B, Sargin M, et al. Surgery of cardiac hydatid cyst. Experience of 39 years. Ann Cardiol Angeiol. (París) 2008;57:58-61.

16. Álvarez R, Alarcón E, González R. Quiste hidatídico retroperitoneal. Rev Chil Cir. 2002:54:536-8.

17. Balci E, Eren N, Eren Ş, Ülkü R. Ruptured hydatid cysts of the lung in children: clinical review and results of surgery. Ann Thorac Surg. 2002;74:889-92.

18. Aribas O, Kanat F, Gormus N, Turk E. Pleural complications of hydatid disease. J Thorac Cardiovasc Surg. 2002;123:492-7.

19. Fatimi S, Naureen S, Moizuddin S, Puri M, Yousuf M, Javed M, et al. Pulmonary hydatidosis: clinical profile and follow up from an endemic region. ANZ J Surg. 2007;77:749-51.

20. Mutaf O, Arikan A, Yazici M, Erdener A, Ozok G. Pulmonary hydatidosis in children. Eur J Pediatr Surg. 1994;4:70-3.

21. Solak H, Ozgen G, Yüskek T, Eren $\mathrm{N}$, Solak N, Kirca N, et al. Surgery in hydatid cyst of the lung. A report of 460 cases. Scand J Thorac Cardiovasc Surg. 1988;22:101-4. 